\section{Falls prevention among older adults at a Nursing home in a northern suburban of Perth in Western Australia}

\author{
Horatius Musembi Malilu, ${ }^{1}$ \\ Deborah Sundin ${ }^{2}$
}

${ }^{1}$ School of Nursing and Midwifery, Aga Khan University, Nairobi, Kenya; ${ }^{2}$ School of Nursing and Midwifery, Edith Cowan University, Perth Western Australia

\section{Abstract}

The objective was to identify factors leading to falls and determine the role of nurses and carers play in falls prevention.

A retrospective audit of the performance of nurses and carers regarding falls prevention among older adults was used. Residents who had a fall during July $2015(\mathrm{n}=25)$ were identified using purposeful sampling. Data for this study were collected from the files of these residents. Data analysis was done by using the Fisher exact test which was appropriate for the collected data.

Residents aged 85-89 years had the most falls (32\%); however, one resident who fell five times in the studied month was aged 90 years. Residents aged 70-74 years had the lowest number of falls $(8 \%)$. Twenty-two (88\%) of the residents who fell were incontinent, $20(80 \%)$ were confused, $19(76 \%)$ were using walking aids and four (16\%) were blind. Only five (20\%) residents who reported falls in the studied month were independent.

Most falls occurred among residents of advanced age, and among those who had incontinence, were confused and failed to use walking aids. Residential home staff should increase vigilance during specific times and monitors closely residents with a high risk of falls.

\section{Introduction}

The global population of people aged 60 years and above will reach two billion by $2050^{1}$ resulting in more age-related diseases and conditions, such as trauma and injuries related to falls. ${ }^{2}$ In 2010 , falls were responsible for $80 \%$ of disabilities resulting from injuries (excluding road traffic accidents). ${ }^{3}$ Older adults are prone to falls because of the changes in their bodies that occur with aging and diseases that occur in old age. ${ }^{4}$
Aging is a complex process that involves many variables (for example, lifestyle, diseases and illnesses, a person's genetic makeup), which interact to influence the manner in which a person ages. Research suggests that there are more falls among those aged 80 years and over than in any other age group. ${ }^{5}$

Falls in older adults can lead to serious complications, including bone fractures, cerebral haematoma, internal organ rupture, and death. ${ }^{6}$ These complications negatively affect the person's wellbeing and their family and increase the financial burden on the government. ${ }^{7}$ However small a fall may be, it can leave a long-lasting mark on an older adult. Psychological and emotional complications are also associated with falls, which may include fear of falling again, loss of confidence, increased anxiety, fear of mobilising and fear of using toilet seats. ${ }^{8}$

The cost of treating injuries due to falls among older adults is high, and most stakeholders in developed countries emphasise falls prevention. ${ }^{9}$ For example, in the United Kingdom, 450,000 people aged $\geq 65$ years were admitted to the hospital as a result of a fall in 2010-2011. ${ }^{9}$ A colossal amount of money is spent yearly on treating and managing fall injuries among older people. From the United States statistics a total of $\$ 31.3$ billion direct medical cost was recorded in $2015^{10}$ and is projected to reach $\$ 100$ billion in the year 2030 (Table 1). ${ }^{11}$ In Australia, around $7 \%$ of total healthcare expenditure is attributed to injuries that are partly associated with the aging population, with patient costs related to older adult trauma being $30 \%$ higher than those for non-older adult patients. ${ }^{12}$ Another Australian study showed that falls were the second largest contributor to the economic burden from injuries with respect to lifetime costs, after motor vehicle injuries. ${ }^{13}$

Without adequate care, older adults are predisposed to multiple falls, especially when carrying out activities of daily living such as accessing the toilet or reaching in a cupboard for items they need. ${ }^{14}$ Other factors such as loss of balance and reduced dietary intake associated with older adults (aged over 65 years) also predispose this group to risk for falls. ${ }^{15}$ According to the Australian Institute of health and welfare (2019), a lot of studies were done on aged population and hospitalization which is more secondary prevention and limited researches on primary prevention. This study emphasize primary prevention in line with the sustainable development goal 3 (target 3.6) that aims to halve the number of preventable deaths and injuries globally by $2030 .{ }^{16,17}$
Correspondence: Horatius Musembi Malilu, School of Nursing and Midwifery, Aga Khan University, P.O. Box 39340-00623, Nairobi, Kenya.

Tel.: +254.726.521.019.

E-mail: horatius.musembi@aku.edu ; malimukombi@gmail.com

Key words: Older adults; aged, residential homes; fall; prevention.

Acknowledgments: the authors acknowledge the residential home facility manager and all staff for their support while undertaking this research.

Funding: this study was undertaken as part of a master's program at Edith Cowan University. No funding was received for the study.

Contributions: HM and DS conceptualised this study. HM developed the research protocol with advice from DS. HM collected data, performed data analysis and wrote the first draft of the manuscript under the supervision of DS. Both HM and DS reviewed the manuscript and approved it for publication.

Conflict of interest: the authors declare no potential conflict of interest.

Received for publication: 24 June 2019.

Revision received: 3 November 2019.

Accepted for publication: 7 November 2019.

This work is licensed under a Creative Commons Attribution-NonCommercial 4.0

International License (CC BY-NC 4.0).

${ }^{\circ}$ Copyright: the Author(s), 2019

Licensee PAGEPress, Italy

Geriatric Care 2019; 5:8358

doi:10.4081/gc.2019.8358

\section{Materials and Methods}

A retrospective audit of the performance of nurses and carers regarding falls prevention among older adults in Nursing home in northern suburb of Perth was conducted. The Nursing home hosted 157 residents, 25 of who had experienced falls in July 2015. The files of residents who had experienced falls were purposefully sampled for the study to help the researchers have an in-depth understanding of the recent trends and determine compliance with relevant policies among the care team. An audit tool to evaluate nurses' and carers' practice in falls prevention was used to retrieve data from the 25 selected files of residents who had experienced falls in July 2015. The audit tool was developed based on issues identified after a discussion with the clinical manager of the studied Nursing 
home. Identified data on the residents' age, cause of the fall (for example, pushed by another resident, forgot to use walking aids, slippery floor), location of the fall (walking, sitting, sleeping), time of the fall (morning, lunch time, afternoon, night), fall witnessed (yes or no), falls prevention measures in place (yes or no) and falls caused by another resident (yes or no) were retrieved. Statistical analysis of retrieved data was carried out using inferential statistics (fisher exact test) which is recommended for smaller data as opposed to large data.

Permission to undertake the study was granted by the Department of Health Sciences and Engineering, Edith Cowan University and the Ethics Committee of the Nursing home. The sampled residents provided consent for the researchers to access their confidential files for data collection.

\section{Results}

The total number of falls reported in the month of July 2015 was thirty-five. Fourteen
(56\%) residents fell once, eight $(32 \%)$ fell twice and one (4\%) had five falls. Residents aged 85-89 years had the most falls $(n=8$, $32 \%$ ) and those aged $70-74$ years had the lowest number of falls $(n=2,8 \%)$. Eighteen residents (72\%) fell while walking, six (24\%) fell while sitting and one (4\%) fell while sleeping (rolling from bed to the floor) this was related to the FRAT score of the residents with a P-value 0.102 possibly related to the small number of residents studied. Residents had high $(\mathrm{n}=22,88 \%)$ and medium risk $(\mathrm{n}=3$, $22 \%$ ) in the falls risk assessment tool score. The majority of falls occurred in the residents' rooms $(\mathrm{n}=22,61.1 \%)$ and dining and activity rooms $(\mathrm{n}=4,11.1 \%)(\mathrm{P}=0.084)$ with a few occurring in other locations.

The falls were related to (more than one aspect applied to the residents being studied); incontinence $(\mathrm{n}=22,88 \%)$, confusion $(\mathrm{n}=20,80 \%)$, not using walking aids $(\mathrm{n}=19$, $76 \%)$, impaired vision $(n=4,16 \%)$ or unidentifiable reasons $(n=5,20 \%)$. Most of the falls happened in the late afternoon (3.01-6.00 pm) (27.8\%), early morning $(6.00-9.00 \mathrm{am})(25 \%)$ or early afternoon $(12.01-3.00 \mathrm{pm}) \quad(19.4 \%) \quad(\mathrm{P}=0.129)$.
Among all the recorded falls, six residents had documented skin tears, but there were no other documented injuries. Regarding caregiver's roles there was $100 \%$ compliance with assessing residents for risk for falls; however, actual prevention was lacking as $80 \%$ of the falls were not witnessed to initiate preventive measures.

\section{Discussion}

The studied Nursing home showed excellent compliance with planning and making residents' nursing care plans available for staff, referring residents after a fall, informing relatives about the fall and updating the resident's Falls Risk Assessment tool score. Most residents who fells had a high risk of falls score. This risk assessment tool has been shown to offer the best fall risk prediction up to $73 \%$ and $75 \%$ in both sensitivity and specificity respectively. ${ }^{18}$ Assessment of the residents was adequately documented In this nursing home and care plan availed in their files

Table 1. Characteristics of residents who experienced falls in July 2015.

\begin{tabular}{|c|c|c|c|c|}
\hline Characteristics & Medium & Hioh & Total & P-value \\
\hline Age, years & & & & \\
\hline 70-79 & $1(0.48)[0.56]$ & $2(2.52)[0.11]$ & 3 & \\
\hline $80-89$ & $2(0.96)[1.13]$ & $4(5.04)[0.21]$ & 6 & \\
\hline 90-99 & $1(2.56)$ [0.95] & 15 (13.44) [0.18] & 16 & \\
\hline & ( & & & 0.2076 \\
\hline
\end{tabular}

\begin{tabular}{lllll} 
Male & 0 & 10 & 10 & 10 \\
Female & 3 & 12 & 15 \\
\hline
\end{tabular}

\section{Location of the falls}

\begin{tabular}{|c|c|c|c|c|}
\hline Residents rooms & $1(2.40)[0.82]$ & $19(17.60)[0.11]$ & 20 & \\
\hline Activity/lounge & $1(0.36)[1.14]$ & $2(2.64)[0.16]$ & 3 & \\
\hline \multirow[t]{2}{*}{ Outside } & $1(0.24)[2.41]$ & $1(1.76)[0.33]$ & 2 & \\
\hline & & & & 0.084 \\
\hline \multicolumn{5}{|c|}{ Timing of the falls } \\
\hline Morning & $1(1.76)[0.33]$ & $10(9.24)[0.06]$ & 11 & \\
\hline Afternoon & $1(1.60)[0.23]$ & $9(8.40)[0.04]$ & 10 & \\
\hline \multirow[t]{2}{*}{ Night time } & $2(0.64)[2.89]$ & $2(3.36)[0.55]$ & 4 & \\
\hline & & & & 0.129 \\
\hline \multicolumn{5}{|c|}{ Activity of residents at time of fall } \\
\hline Sleeping/sitting & $2(0.80)[1.80]$ & $3(4.20)[0.34]$ & 5 & \\
\hline Walking & $2(3.20)[0.45]$ & $18(16.80)[0.09]$ & 20 & \\
\hline
\end{tabular}


In this Nursing home, most of the activities (waking up, bathing, exercise, and meals) were routine and timed making the residents get used to them. Most falls occurred in the late afternoon when residents were supposed to report for routine activities or early morning during scheduled time for toileting and bathing. Therefore, unavailability of caregivers to support and monitor residents as they perform these activities on their own, led to unwitnessed falls. ${ }^{19}$

Our study showed that six out of every ten residents who fell were females. Many studies have shown that females have fewer muscle compositions and increased muscle loss with aging compared to males. ${ }^{20}$ This study also found that the risk of falls and multiple falls increased with advancing age. These findings are similar to that of a study in the United States where the number of falls increased from $9.9 \%$ to $13.5 \%$ among $65-74$ years old and 85 years old, respectively. ${ }^{21}$ Advanced age is associated with physical changes such as reduced body strength and muscle mass wasting. ${ }^{22,23}$

Similar to other studies, we found that a majority of the residents who fell were incontinent, confused, blind and failed to use walking aids. ${ }^{24}$ Poor use or complete failure to use the walking aids like walking sticks, wheelchairs and other assistive devices, and comorbidities such as dementia have been shown to contribute to a high number of falls and injuries among old adults. ${ }^{4}$ A majority of the falls occurred in the residents' room, which is similar to the documented location of falls in other studies. $^{25}$ The residents spend more time in their rooms than in any other place in Nursing homes. ${ }^{25}$

The risk factors identified in this study are consistent with other research findings from other parts of the world; where it is a combination of many factors that result in falls among older adults. ${ }^{26}$

Falls result from multiple causes and factors including person-specific and environmental factors. ${ }^{4,27}$ In this study, falls were more prevalent among females and the aged residents and resulted from incontinence, confusion, visual impairment, and failure to use or improper use of assistive walking devices and unavailability of caregivers.

\section{Limitations}

This study used cross-sectional design hence causation could not be inferred. The study had a small sample size limiting assessment of risk factors and generalisation of the findings to Western Australia. This study could not also ascertain the reasons for the patterns of falls.

\section{Conclusions}

Falls among older adults continues to increase among those living in aged care homes. Despite residents being assessed for risks of falls, factors such memory impairment, incontinence, and visual impairment were among the causes of falls among older adults. Caregivers need to be sensitised to be more vigilant and alert to avert falls among residents; for example, by constantly reminding them to use walking aids, placing them within reach and assisting them with their daily routines. The results of this research are key in predicting the moments and time when aged residents are likely to fall and give a clear insight into primary prevention rather than secondary and tertiary prevention.

\section{References}

1. United Nations DoEaSA. World population ageing 2017: highlights. United Nations New York; 2017.

2. Michaud M, Balardy L, Moulis G, et al. Proinflammatory cytokines, aging, and age-related diseases. J Am Med Direct Assoc 2013;14:877-82.

3. Stewart Williams J, Kowal P, Hestekin $\mathrm{H}$, et al. Prevalence, risk factors and disability associated with fall-related injury in older adults in low- and middleincomecountries: results from the WHO Study on global AGEing and adult health (SAGE). BMC Med 2015;13:147.

4. Ambrose AF, Paul G, Hausdorff JM. Risk factors for falls among older adults: a review of the literature. Maturitas 2013;75:51-61.

5. Garatachea N, Lucia A. Genes, physical fitness and ageing. Ageing Res Rev 2013;12:90-102.

6. Poll MA, Hoffmeister ACM, Tier CG, Santos SSC. Occurrence of fall in elderly hospitalizations. Cienc Cuid Saude 2014;13:447-54.

7. Vivian W, Shixun G. Family Caregiving and Impact on Caregiver Mental Health: A Study in Shanghai. Aging in China: Springer; 2012. p. 187-207.

8. Huang AR, Mallet L, Rochefort CM, et al. Medication-related falls in the elderly. Drugs Aging 2012;29:359-76.

9. Duffy A. The assessment and management of falls in residential care settings. Br J Nurs 2013;22:259-63.

10. Burns ER, Stevens JA, Lee R. The direct costs of fatal and non-fatal falls among older adults - United States. J Safety Res 2016;58:99-103.
11. Houry D, Florence C, Baldwin G, et al. The CDC Injury Center's response to the growing public health problem of falls among older adults. Am J Lifestyle Med 2016;10:74-7.

12. Dinh MM, McNamara K, Bein KJ, et al. Effect of the elderly and increasing injury severity on acute hospital resource utilization in a cohort of inner city trauma patients. ANZ J Surg 2013; 83:60-4.

13. Heinrich S, Rapp K, Rissmann U, et al. Cost of falls in old age: a systematic review. Osteop Int 2010;21:891-902.

14. Urquhart Wilbert W. The Effectiveness of a Fall Prevention/Management Program In Reducing Patient Falls: A Retrospective Study. JOCEPS J Chi Eta Phi Sorority 2013;57:1.

15. Albert SM, King J, Boudreau R, et al. Primary prevention of falls: effectiveness of a statewide program. Am J Public Health 2014;104:e77-e84.

16. Robert KW, Parris TM, Leiserowitz AA. What is sustainable development? Goals, indicators, values, and practice. Environ Sci Policy Sustain Develop 2005;47:8-21.

17. Mokdad AH, Forouzanfar MH, Daoud F, et al. Global burden of diseases, injuries, and risk factors for young people's health during 1990-2013: a systematic analysis for the Global Burden of Disease Study 2013. Lancet 2016;387:2383-401.

18. da Costa BR, Rutjes AWS, Mendy A, et al. Can falls risk prediction tools correctly identify fall-prone elderly rehabilitation inpatients? A systematic review and metaanalysis. PLoS One. 2012;7:e41061.

19. Gray-Miceli D, Quigley PA. Fall prevention: assessment, diagnoses, and intervention strategies. Evid-Based Geriatr Nurs Protocols Best Pract 2012;4.

20. Chang VC, Do MT. Risk factors for falls among seniors: implications of gender. Am J Epidemiol 2015;181:521-31.

21. Bergen G. Falls and fall injuries among adults aged $\geq 65$ years - United States, 2014. MMWR Morbid Mortal Weekly Rep 2016;65.

22. Kalyani RR, Corriere M, Ferrucci L. Age-related and disease-related muscle loss: the effect of diabetes, obesity, and other diseases. Lancet Diabet Endocrinol 2014;2:819-29.

23. Grundstrom AC, Guse CE, Layde PM. Risk factors for falls and fall-related injuries in adults 85 years of age and older. Archiv Gerontol Geriatr 2012;54: 421-8.

24. Gale CR, Cooper C, Aihie Sayer A. Prevalence and risk factors for falls in older men and women: The English Longitudinal Study of Ageing. Age Ageing 2016;45:789-94. 
25. Stevens JA, Mahoney JE, Ehrenreich H. 26. Dionyssiotis Y. Analyzing the problem Circumstances and outcomes of falls among high risk community-dwelling older adults. Injury Epidemiol 2014;1:5. of falls among older people. Int J Gener Med 2012;5:805.

27. Landi F, Liperoti R, Russo A, et al.
Sarcopenia as a risk factor for falls in elderly individuals: results from the ilSIRENTE study. Clin Nutr 2012;31: 652-8. 